\title{
Overexpression of Merlin in B16F10 mouse melanoma cells reduces their metastatic activity: Role of the cell surface heparan sulfate glycosaminoglycans
}

\author{
ZOYA GALCHEVA-GARGOVA ${ }^{1}$, NATALIA ZHIDKOVA ${ }^{2}$, SARA GEISLER ${ }^{3}$, \\ JENNIFER OZUG $^{1}$, STEVE WUDYKA ${ }^{1}$, NUR SIBEL GUNAY ${ }^{1}$, YI WEI QI ${ }^{1}$, \\ ZACHARY SHRIVER $^{1}$ and GANESH VENKATARAMAN ${ }^{1}$ \\ ${ }^{1}$ Momenta Pharmaceuticals, Inc.; ${ }^{2}$ Biogen IDEC, Cambridge, MA 02142; \\ ${ }^{3}$ Novartis Pharmaceuticals, Cambridge, MA 02139, USA
}

Received December 24, 2007; Accepted February 18, 2008

\begin{abstract}
Merlin, the protein product of the neurofibromatosis type 2 gene (NF2) acts as a tumor suppressor in mice and humans. In this study, melanoma B16F10 cells were engineered to overexpress the NF2 gene by establishing stable transductants. A cell line overexpressing Merlin (B16F10-M) was generated. When compared to the parental cells, the B16F10-M cells demonstrated differences in their cell surface organization. The overexpressing strain changed its ability to grow in soft agar as well as its cell motility properties. $\mathrm{B} 16 \mathrm{~F} 10-\mathrm{M}$ cells were then examined in the in vivo mouse melanoma tumor growth and tumor metastasis models. While tumor growth was marginally affected, the presence of increased Merlin severely reduced the metastastatic ability of the cells. When isolated using specific enzymes with distinct substrate specificity, the cell surface heparan sulfate glycosaminoglycans (HSGAGs) from the overexpressing B16F10-M cells, inhibited the metastatic properties of the parental B16F10 cells. The results obtained provide a causal link between the reorganization/changes to the cell surface HSGAGs by the overexpression of Merlin and the inhibition of the metastatic activity of the mouse melanoma B16F10 cells in vivo.
\end{abstract}

\section{Introduction}

The neurofibromatosis type 2 gene (NF2) encodes Merlin, an ezrin-radixin-moesin-(ERM)-related protein, which acts as a tumor suppressor whose absence results in the occurrence of multiple tumors of the nervous system, particularly schwan-

Correspondence to: Dr Zoya Galcheva-Gargova, Momenta Pharmaceuticals, Inc., 675 West Kendall Street, Cambridge, MA 02142, USA

E-mail: zoya@momentapharma.com

Key words: Merlin, metastasis, heparan sulfate glycosamionoglycans nomas and meningliomas. The NF2 gene was cloned more then 10 years ago but its function still remains a focus in a number of laboratories. Localized in membrane ruffles and along cell boundaries, Merlin links the membrane's associated proteins and the actin cytoskeleton, and provides a function in receiving and interpreting extracellular signals. Initial studies of the function of Merlin, like other tumor suppressor genes, demonstrated that its overexpression can block cell proliferation and oncogene-induced transformation $(1,2)$. Later it was shown that indeed Merlin negatively regulates cyclin D1 levels, leading to cell cycle arrest upon overexpression (3). However because of its localization to the membrane cytoskeleton interface, Merlin functions as a novel type of tumor suppressor by regulating the linkage between the membrane's associated proteins and the cytoplasm. The latest data suggest that Merlin may coordinate the processes of growth factor signaling and cell adhesion, events of major importance for tumor growth and metastasis $(4,5)$.

One of the major components of the extracellular matrix are the glycans. Glycans are assemblies of sugars that exist either in free form or in covalent complexes with proteins and lipids. The glycosaminoglycans (GAGs) are present as free polysaccharides as the hyaluronan or as parts of proteoglycans, such as heparan sulfate (HS) and chondroitin sulfate. Most classes of glycans consist either as membrane-bound glycan-conjugates or as secreted molecules that become an integral part of the extracellular matrix (ECM). The localization of the glycans suggests their function in mediating cell adhesion and motility, as well as intracellular signaling events crucial for tumor progression.

There is mounting clinical evidence suggesting correlation between the changes in glycosylation in the cell surface and poor prognosis in cancer, but understanding the role of specific glycans in the biology of any tumor is still a considerable challenge. The difficulties include the microheterogeneity of glycosylation on one hand and the overall complexity of the factors leading to malignant transformation on the other. There is still no definitive conclusion concerning the causal link between carbohydrates and the malignant phenotype. It has been hypothesized that the glycan coat can protect tumor cells from immune surveillance, and that the 
cell surface specific glycans mediate adhesion to distant sites and promote tumor cell metastasis $(6,7)$.

In the present study we overexpressed Merlin in the mouse B16F10 melanoma cells. Several properties such as motility contact inhibition of growth, as well as immunochemical characteristics of the transformed cell line are presented. The results of the experiments using the NF2 overexpressing cells in the in vivo tumor growth and tumor metastasis models, as well as the effect of the isolated fraction of cell surface GAGs on the metastasis of the parental cells, suggest that Merlin overexpression changes/redistributes the cell surface HSGAGs and that these specific changes of glycosaminoglycan organization influence the oncogenic potential of the cells.

\section{Materials and methods}

Cell lines. Mouse melanoma cell line B16F10 was obtained from ATCC. The cells were cultured in Dulbecco's modified Eagle's medium (DMEM) with $4 \mathrm{mM}$ L-glutamine adjusted to contain $1.5 \mathrm{~g} / \mathrm{l}$ sodium bicarbonate and $4.5 \mathrm{~g} / \mathrm{l}$ glucose, and $10 \%$ fetal bovine serum, as required by the ATCC instructions. For animal injections, the cells were grown to 80-90\% confluency, trypsinized, and washed 3 times with phosphate buffered saline (PBS), each time using low speed centrifugation $\left(800 \mathrm{x} \mathrm{g}\right.$ ) at $4^{\circ} \mathrm{C}$ for $5 \mathrm{~min}$. After the last wash the cells were counted (Beckman Coulter) and diluted to the required density for injection into the mice.

Generation of B16F10-M cells by stable transfections. The Merlin gene was obtained by PCR of a mouse embryo library (17-day Embryo Quick-Clone cDNA, BD Biosciences) with primers specific for the sequence: Merlin forward primer 5'GGATCCATGGCCGGAGCCATCGCTTC3', and Merlin reverse primer 5'CTTAAGGAGTTCTTCAAAGAAGGCCA3' [bases 1-2241 of the NF2 isoform I and cloned into BamH1/ EcoRI sites of the pcDNA3.1(+) (Invitrogen)]. Transfections were carried out using Lipofectamine (Invitrogen) according to the instructions provided by the manufacturer. In brief, $25 \mu \mathrm{g}$ of DNA and $50 \mu \mathrm{l}$ of Lipofectamine were mixed and incubated for $20 \mathrm{~min}$ at room temperature. Cells, 80-90\% confluent, in 100-mm dishes were washed with Opti-Mem media and the DNA/Lipofectamine mixture was added. After an overnight incubation, the medium in each plate was replaced with the selective media DMEM/10\% FBS supplemented with G-418 (Geneticin, Gibco), a neomycin analog at $800 \mu \mathrm{g} / \mathrm{ml}$. The selection was continued for 2 weeks, by replacing the media with Geneticin every 3 days.

Total cell lysates were prepared and protein concentration was measured using a Pierce BCA Protein Assay Kit. Western blots were performed according to the ECL detection reagents booklet from Amersham, GE Healthcare.

Antibodies. Merlin-specific antibodies: rabbit polyclonal (Novus Biologicals, Inc. ab2478) 1:1000 dilution for Western blotting, rabbit polyclonal for immunostaining (Santa Cruz, sc332, 1:50 dilution); vinculin-specific antibody, monoclonal anti-human (Sigma, V9131, 1:400); HSP 3G10 antibody, monoclonal anti-human heparan sulfate antibody (Seikagaku, 370260-1); Cy2 goat anti-rabbit IgG and Cy5 goat-antimouse IgG were used as secondary antibodies (Jackson
ImmunoResearch Laboratories, Inc., 11-227-0030, and 115177-0030 respectively).

Immunostaining. The procedures used were performed as recommended by the antibody manufacturers. Briefly, cells were plated onto 4-well chamber slides and grown in complete media overnight. For staining with the anti-Merlin antibody the cells were fixed with $4 \%$ paraformaldehyde in PBS for $20 \mathrm{~min}$, permeabilized $10 \mathrm{~min}$ with $0.2 \%$ Triton $\mathrm{X}-100$, blocked in $1 \%$ BSA-PBS for $15 \mathrm{~min}$, incubated for $1 \mathrm{~h}$ with the primary antibody diluted in BSA-PBS, and then incubated for $30 \mathrm{~min}$ with fluorescently-labeled secondary antibody. Slides were mounted in mounting solution, and analyzed by immunofluorescent microscopy. For staining with the anti-vinculin antibody, the slides were first placed in ice-cold methanol for $5 \mathrm{~min}$ and stained as described above. The blocking step was performed in $3 \%$ normal goat serum in PBS. Prior to staining with the HSP 3G10 antibody and after fixing the cells in $4 \%$ formaldehyde for $10 \mathrm{~min}$, the cells were rinsed with $0.5 \%$ Triton $\mathrm{X}-100$ for $3 \mathrm{~min}$ and treated with heparinase III $(0.5$ units $/ \mathrm{ml})$ for $1 \mathrm{~h}$ at $37^{\circ} \mathrm{C}$. Next the samples were washed and blocked as described above. Images were taken on an inverted immunofluorescent Olympus IX51 microscope.

Mice. Male C57BL/6 mice, aged 6-8 weeks (Charles River Laboratories, Inc.), were selected for these experiments. The animals were housed 3-4 mice per cage in a temperature- and humidity-controlled room. The mice were kept in a 12-h circadian cycle and provided access to food and water ad libitum. All experimental procedures were performed in accordance with federal, local, and institutional guidelines.

Tumor cell implantation. Prior to the experiment, the animals were randomly assigned to receive one of two different cancer cell types: B16F10 or B16F10-M. On the first day of the experiment, tumor cell suspensions $\left(5 \times 10^{5}\right.$ in $\left.0.1 \mathrm{ml} \mathrm{PBS}\right)$ were injected subcutaneously into the right flank of each mouse. Following implantation, tumors were monitored daily for palpable tumor masses, and tumor length and width were measured with digital calipers. Tumor volumes were defined using the formula: length $\mathrm{x}$ (width) ${ }^{2} \mathrm{x} 0.532$; with length considered the longest diameter, width considered the shortest diameter of the tumor mass, and 0.532 included as the conversion constant. At 19 days post-implantation, mice from the B16F10 and B16F10-M groups $(\mathrm{n}=8)$ were sacrificed via $\mathrm{CO}_{2}$ asphyxiation in accordance with IACUC guidelines, and the tumors were removed.

Tumor cell metastasis. For the metastatic model, mice were randomly assigned $(n=8)$ to receive an intravenous injection of $2 \times 10^{5} \mathrm{~B} 16 \mathrm{~F} 10$ cells, B16F10-M cells, or the same volume of saline. The HSGAGs fractions isolated and prepared as described bellow (from control and Merlin-overexpressing cells) were added in equal amounts to the $2 \times 10^{5}$ cell suspension prior to injection. The mice were monitored for 14 days and then euthanized via $\mathrm{CO}_{2}$ asphyxiation according to IACUC guidelines. The lungs were immediately excised. Visible lung cancer nodules were examined through a dissecting light-microscope at x 2.5 and the nodules counted 
with the use of a hand-held counter (8). Lungs were dissected when necessary to ensure accurate nodule counts.

Statistics. Data are presented as mean \pm SE. Comparisons between groups were made via the two-tailed Student's t-test, with significance set at $\mathrm{p}<0.05$.

Cell motility assay. Cell motility was assessed in a wound healing assay essentially as described (9). Briefly, cells were grown up to $90 \%$ confluency in 12 -well plates in $10 \% \mathrm{FBS} /$ DMEM. Five hours after changing the media to $2 \% \mathrm{FBS} /$ DMEM, a scratch was introduced in the middle of the cells using a yellow pipette tip. A scratch was introduced in 3 wells for each type of cell plated. Three washes were performed with DMEM and a picture of each well was taken, with a second picture taken $12 \mathrm{~h}$ later. The plates were examined on an Olympus IX51 microscope at x400 magnification and three separate measurements of the area were performed for each well, with IP3Lab software used to calculate the motility. The ratio of the average cell-free area after $12 \mathrm{~h}$ was plotted to the initial area of the scratch.

Soft agar colony formation assays. For the anchorageindependent growth assay, 60-mm dishes were covered with $0.5 \%$ agar DMEM with $10 \%$ FBS and the two types of cells $\left(5 \times 10^{3}\right.$ cells/well) were plated in $0.25 \%$ agar. The cells were allowed to grow in soft agar for up to 2 weeks at $37^{\circ} \mathrm{C}$.

Anchorage-independent growth was assessed by scoring the colonies using an inverted microscope.

Cell surface GAG isolation and purification. B16F10 and B16F10-M cells were grown to $75 \%$ confluency, washed with PBS, and treated with $8 \mathrm{ml}$ of either PBS, heparinase I $(10 \mathrm{~g} / \mathrm{ml})$, or heparinase III $(25 \mu \mathrm{g} / \mathrm{ml})$ in PBS $\left(\mathrm{Ca}^{2+}\right.$ and $\mathrm{Mg}^{2+}$ free) for each $\mathrm{T} 225$ plate, for $30 \mathrm{~min}$ at $37^{\circ} \mathrm{C}$. The microscopic analysis of the enzyme-treated cells revealed no differences when compare to the cells treated with PBS alone: the cells remained attached to the plate surface. The supernatants containing the fraction of the digested material were cleared by centrifugation for $10 \mathrm{~min}$ at $4500 \mathrm{x} \mathrm{g}$, boiled for $15 \mathrm{~min}$ and filtered $(0.2 \mu \mathrm{m}$-filter $)$. Next, the samples were reconstituted after lyophilisation in $1.5 \mathrm{ml}$ of $50 \mathrm{mM}$ Tris $\mathrm{HCl} \mathrm{pH} 5.5$ and centrifuged for $5 \mathrm{~min}$ at $10,000 \mathrm{rpm}$. Supernatant $(1 \mathrm{ml})$ was injected via a $5-\mathrm{ml}$ superloop onto a 1.8-ml HR 10/2 column containing Source ${ }^{\mathrm{TM}}$ 15Q resin. The column was equilibrated with $50 \mathrm{mM}$ Tris $\mathrm{HCl} \mathrm{pH} 5.5$ (mobile phase A) at $1 \mathrm{ml} / \mathrm{min}$ for a total of 12 column volumes. B16F10 and B16F10-M samples were fractionated with a step gradient which incrementally increased the percentage of mobile phase B $(50 \mathrm{mM}$ Tris $\mathrm{HCl} / 1 \mathrm{M}$ sodium chloride, $\mathrm{pH} 5.5$ ) by $20 \%$ over $20 \mathrm{~min}$. The UV signal was monitored at $232 \mathrm{~nm}$ and fractions were collected every $1.4 \mathrm{ml}$. The pooled fractions were buffer exchanged into $40 \mathrm{mM}$ ammonium acetate using a 20-ml HR 16 column containing G-10 Sephadex resin. The UV signal was monitored at $232 \mathrm{~nm}$ and fractions were collected every $2.5 \mathrm{ml}$. The bufferexchanged pooled fractions from B16F10 and B16F10-M were frozen and lyophilized. Final gravimetric weights were obtained and a metahydroxybiphenyl assay was performed to determine a yield based on uronic acid content.
A

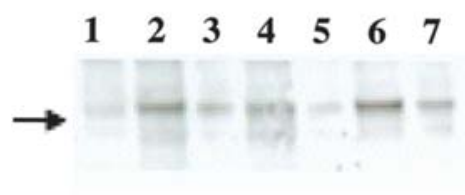

B 1 2
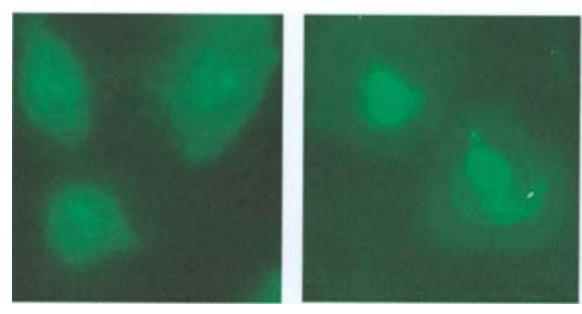

Figure 1. Overexpression of Merlin in B16F10 cells. (A) Western blot analysis of cell lysates prepared from selected different stable clones (lanes 2-6), and (lane 1) lysate from the parental cells (the arrow indicates the $75-\mathrm{kD}$ size marker). (B) Immunostaining of the control cells (1) and the B16F10-M cells (2) with a Merlin specific antibody (x400 magnifications).

The procedure yielded reproducible amounts of HSGAGS for the in vivo studies.

\section{Results}

B16F10-M - a cell line overexpressing Merlin. The introduction of additional copies of NF2 in the genome of B16F10 was created by stable transfection. The Merlin gene was obtained by PCR of a mouse embryo library with primers specific for the sequence and inserted in a commercially available vector (pcDNA3.1). As a control, a mix of stable clones was generated using the same plasmid with CAT gene as an insert. It was demonstrated in a previous study (8) that the oncogenic characteristics of the CAT expressing cell line when tested in our in vivo models and in vitro assays, were the same as the parental strain.

Several positive clones were picked, grown and characterized using a Western blot probed with a Merlinspecific antibody (Fig. 1A). A more intensive signal for the NF2 antibody was demonstrated in the transformants, although the amount of total protein loaded was the same in all lanes. It is interesting to note that the degree of overexpression of Merlin was moderately increased (3-4 times) when compared with control cells. For further experiments, one of the tested clones was picked (Fig. 1A, lane 6) and referred to as B16F10-M. The overexpression of NF2 was also confirmed by immunostaining the cells with a Merlinspecific antibody (Fig. 1B). A more intensive fluorescence signal from the B16F10-M cells upon comparison with the control cell line was demonstrated, confirming the increased expression of Merlin molecules in the transfected cells.

B16F10-M cells demonstrate a distinct pattern of staining with antibodies specific for the cell surface. Merlin is 
A
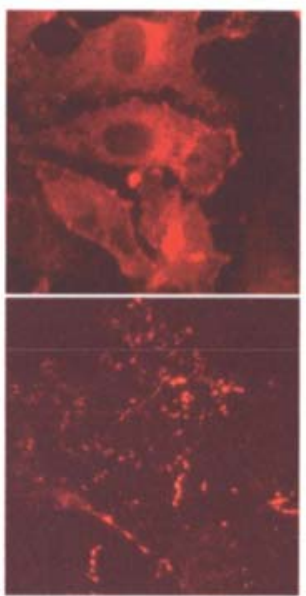

C
B
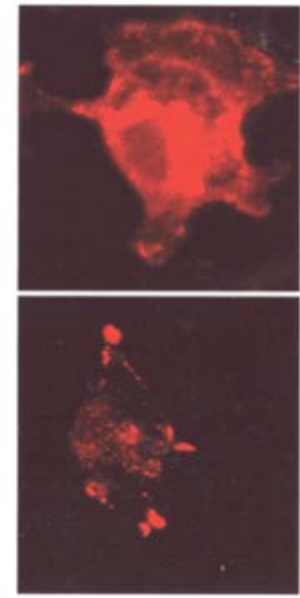

D
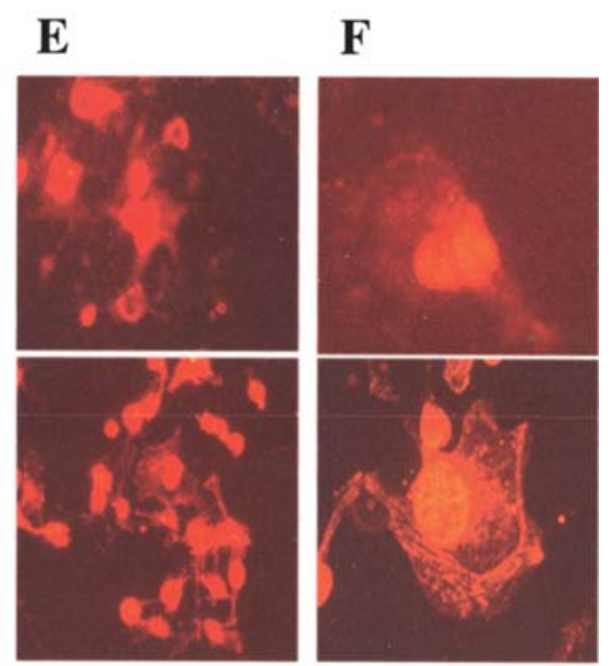

G

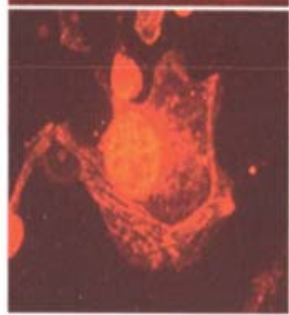

H

Figure 2. Immunostaining experiments. Changes in the cell surface organization of the B16F10-M cells upon staining with an vinculin-specific antibody (C,D) and with the HS3G10 antibody $(\mathrm{G}, \mathrm{H})$ when compared with the control B16F10 cells [(A,B) and (E,F) respectively]. Magnification: $\mathrm{x} 400(\mathrm{~B}, \mathrm{D}, \mathrm{F}$ and H) and $\mathrm{x} 200(\mathrm{~A}, \mathrm{C}, \mathrm{E}$ and $\mathrm{G})$.

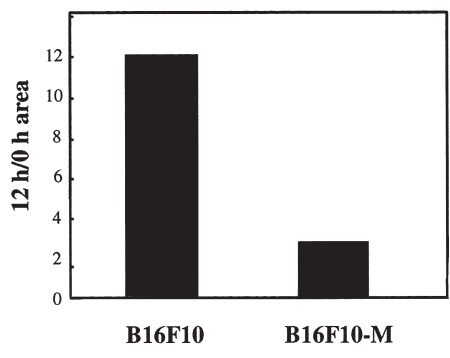

Figure 3. Cell motility results of the wound healing assay. The ratio of the area measured $12 \mathrm{~h}$ after introducing the scratch was plotted to the initial scratch area The results for B16F10 cells and the Merlin-overexpressing cell line are presented.

positioned under the cellular membrane and is thought to be involved in transmitting signals from the extracellular matrix. In early studies it was demonstrated that increased expression of NF2 in schwannoma cell lines results in transient alteration in F-actin organization, cell spreading and cell attachment (10). Furthermore it was shown that the major cellular consequence of NF2 deficiency in primary cells is an inability to undergo contact-dependent growth arrest and to form stable cadherin-containing cell to cell junctions (11). Recently it was suggested that Merlin coordinates the process of adherent junction (12). In the case of the mouse melanoma B16F10 cell line it was important to investigate whether B16F10-M cells would demonstrate a change in the pattern of focal adhesion, a process very important for the malignant properties of cancer cells. In mammalian cells, vinculin is a membrane-cytoskeletal protein in focal adhesion plaques that is involved in the linkage of integrin adhesion molecules to the actin cytoskeleton. The results of immunostaining the cells with vinculin-specific antibodies are presented in Fig. 2A-D. Our results suggest that the upregulation of Merlin morphologically alters the pattern of distribution of the focal contact points; they are punctuated to a higher degree when compared
A

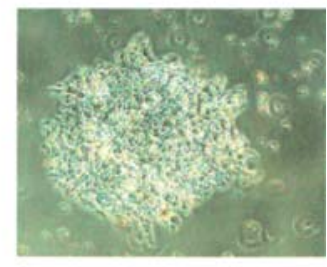

B

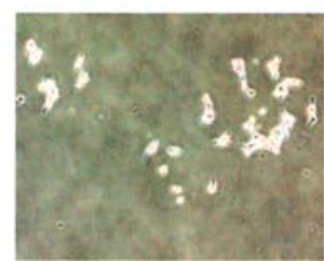

Figure 4. Soft agar assay. A phase-contrast photograph of a representative field of the plates with colonies grown in soft agar control B16F10 (A) and B16F10-M cells (B).

with the control cell line, indicative of a higher adhesive phenotype in the B16F10-M cells.

The HSGAGs are a major component of the extracellular matrix and presumably are involved in the different stages of tumor cell migration and invasion (13). The $3 \mathrm{G} 10$ antibody recognizes heparan sulfate neo-epitope generated by digesting the HSGAGs. The unsaturated uronic acid that is present at the non-reducing end of the fragment created by the enzyme is critical for the reactivity of this antibody. Immunostaining with the 3G10 antibody revealed morphological differences between the control cells and the B16F10-M cells; the shape and distribution of the heparan sulfate-like glycosaminoglycans was different (Fig. 2E-H).

B16F10-M cells display differences in a motility assay. As a consequence of the reorganization of the cell surface constituents (adhesion points and HSGAGs), the possibility 

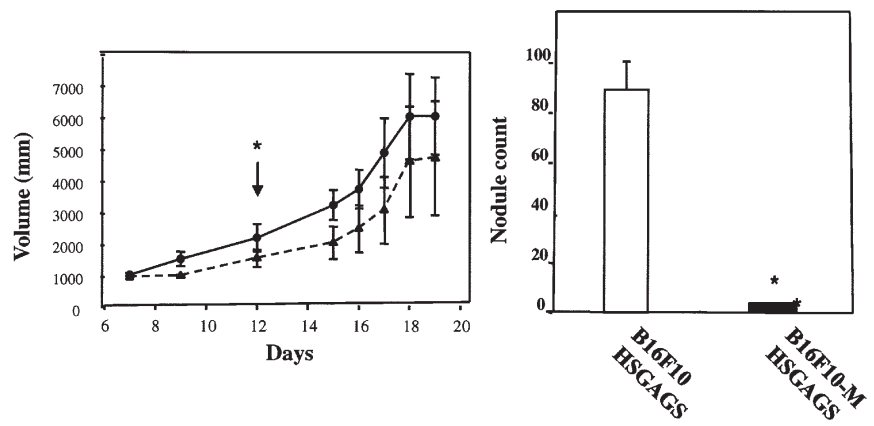

Figure 5. Effect of increased expression of NF2 in the tumor growth and tumor metastasis models. (A) Tumor growth. A total of 8 animals were injected with B16F10 cells (solid line) or with B16F10-M cells (dotted line), and the tumor volumes were measured at different days as a parameter for the tumor growth as described in Materials and methods ("significant difference $\mathrm{p}<0.05$ ). (B) Tumor metastasis. The lungs of the two groups of animals $(n=8)$ injected in the tail vein with one of the two types of cells were isolated on day 15 and the nodules were counted as described in Materials and methods.

of changes in the cell motility in the NF2-overexpressing cells was next explored. The wound healing assay is an in vitro assay in which an artificial space is created by introducing a scratch in a plate with growing cells. Next, after a fixed period of time the cell-free area is plotted against the initial scratch area as an index of cell motility: a larger ratio is indicative of an increased speed at which the cells repopulate the free space. The results in Fig. 3 indicate that the ability of B16F10-M cells to migrate was severely impaired ( 6 times), as compared to the control cells, a result in accordance with reduced oncogenic properties and with changes in the cell surface organization.

Anchorage-independent growth. One of the characteristics of malignant metastatic cells is their ability to grow and form colonies in soft agar. When plated in the soft agar as described in Materials and methods, the B16F10 cells form sizable colonies, as they are highly transformed cells. In contrast, under the same experimental conditions, the B16F10-M cells did not grow as well; the colonies observed were smaller (Fig. 4). These results indicate that the increased expression of $\mathrm{NF} 2$ in the B16F10 cells impaired the anchorage-independent growth.

Tumor growth and tumor metastasis of B16F10-M cells. The results obtained (changes in the focal adhesion patterns, GAGs distribution, motility and soft agar growth) suggested that the tumor properties of the Merlin-B16F10 cells might have changed. To test this possibility, we performed experiments in vivo using the tumor growth melanoma model and the induced tumor metastasis model.

After injection of the two groups of animals with either B16F10 cells or B16F10-M cells, tumor volume was measured as outlined in Materials and methods. The results in Fig. 5A demonstrate that the increased expression of the NF2 gene in the animals injected with $\mathrm{B} 16 \mathrm{~F} 10-\mathrm{M}$ reduced the tumor growth, as measured by the tumor volume. It is important to note that the most pronounced effect on tumor growth
A

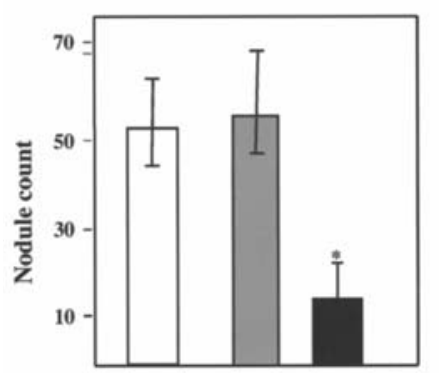

Figure 6. Effect of cell surface HSGAG isolates on the metastasis of the B16F10 cells. (A) Digestion with HepIII was used to prepare cell surface material from B16F10 cells as well as from B16F10-M cells. When injected with the control cells, the HSGAG material prepared from the B16F10-M cells dramatically inhibited the metastasis (black bar) as compared to the addition of the same amount of material prepared from the parental cell line (grey bar). The number of metastases in the animals injected with the B16F10 cells (no cell surface material added) is also presented (white bar). (B) Comparison of the addition of cell surface material isolated from the cells overexpressing the NF2 gene and from the control cells, using enzymes with different HSGAG specificity. Using HepI, cell surface fraction was prepared from both B16F10 and B16F10-M cells. This was added to the control cells and injected into the animals, and the nodules were counted after 15 days. The graph shows B16F10 cells: without added material (white bar); with the addition of the isolate from the control cells (striped bar); and with the addition of the the cell surface fraction from the cells overexpressing Merlin (grey bar). The fraction obtained after digestion of the B16F10-M cells with HepIII was also added to the B16F10 cells (black bar). The amount of added material was the same in all experimental groups and PBS was added in the same volume in the control group.

reduction was observed at the early stages of development of the subcutaneous tumor, when a statistically significant difference was observed in comparison with the size of the tumors injected with the control B16F10 cells.

The conventional way to assess the degree of metastasis is to isolate the lungs as the primary organ invaded by the melanoma cells. As the B16F10 cells produce the pigment melanin, the degree of metastasis is validated by counting the black spots in the lungs of each animal. Lungs were isolated 15 days after injection with B16F10 cells or B16F10-M cells. The results are presented in Fig. 5B and show that the presence of increased Merlin protein almost completely eradicated the metastasis. The inhibition of the metastastatic properties of the B16F10-M cells correlates with both the morphological changes and the motility results that were obtained in vitro.

One possible consequence of the overexpression of Merlin might be a difference in the organization/exposure of the cell surface HSGAGs, which may result in the lost ability of the cells to attach and invade the lung epithelium. To test this hypothesis we prepared a cell surface fraction of the heparan sulfate glycosaminoglycans by digesting the cells with specific enzymes. It was demonstrated in a previous study that there are bioactive sequences in the tumor cell surface glycosaminoglicans of the melanoma cells that influence tumor growth and tumor metastasis (14). Heparinase I (HepI) and heparinase III (HepIII) are enzymes with distinct HSGAG substrate specificities, the former cleaving at highly sulfated regions, while the latter cleave at the under-sulfated regions in polysaccharide chains. The possibility of investigating the influence of the products generated using these two enzymes was tested next. 
Firstly, cell surface HSGAGs were isolated with HepIII from the B16F10 cells and from the B16F10-M cells as described in Materials and methods. Next, the effect of the obtained cell surface material was tested in vivo. The experiment included 3 groups of animals: the first group was injected with B16F10 cells alone, the second group was injected with B16F10 cell surface isolate added to the B16F10 cells, and the third group was injected with the cell surface isolate from the B16F10-M cells in addition to the B16F10 cells. In each group the number of animals tested was the same $(n=8)$ as was the amount of cell surface material injected $(25 \mu \mathrm{g} / \mathrm{kg})$. The number of nodules in the lungs was counted as usual to estimate the degree of metastasis. While the addition of material isolated from the control cells had no effect on the metastatic properties of the B16F10 cells, the cell surface HSGAG fraction from the B $16 \mathrm{~F} 10-\mathrm{M}$ cells drastically inhibited the formation of metastasis (Fig. 6A). Next, a HepI cell surface isolate was prepared from B16F10-M cells and all preparations were tested in the metastasis model as described above. The results presented in Fig. 6B indicate that the addition of cell surface heparan sulfate glycosaminoglycan fraction from the Merlin-overexpressing cells inhibited the formation of metastasis. It is noteworthy that the material obtained by digestion with HepIII inhibited the formation of metastasis to a higher degree as compared to the effect of the cell surface fraction obtained using HepI. As the digestion with the two heparinases used in the described experiments would solubilize the most accessible structures of the cell surface HSGAGs, the results obtained suggest that the cell surface heparan sulfate glycosaminoglycans in melanoma cells with increased expression of NF2 had acquired some differences in their structures/organization that are important for the ability to attach and invade the lungs of the injected animals. Thus, when added to the highly metastatic B16F10 cells, the process of metastasis was strongly impaired.

\section{Discussion}

In the present study, the importance of the expression of Merlin, the product of NF2 gene, was investigated in the B16F10 mouse melanoma cell line. Although detectable by standard procedures, the amount of Merlin protein in these cells is low, thus allowing the engineering of stable transformants overexpressing the NF2 gene. The properties of the transformed cell line B16F10-M were studied using both in vitro experiments and two animal models: tumor growth and induced tumor metastasis models.

Somatic loss of Merlin is causal to tumor development in mice and humans. Furthermore, the analysis of the cellular and molecular consequences of NF2 deficiency in primary cells has demonstrated that Merlin molecules are necessary for the ability of cells to undergo contact-dependent growth arrest and to form stable cadherin-containing cell to cell junctions, pointing to the function of Merlin as a tumor and metastasis suppressor by controlling cell to cell contacts (11).

There are reports in the literature on the role of Merlin in suppressing cell growth when overexpressed in NIH-3T3 fibroblasts and in rat schwannoma cells. The overexpression of wild-type Merlin resulted in transient alteration in F-actin organization, cell spreading and cell attachment. In addition, the migration properties of the overexpressing cells were also impaired $(4,12)$.

The results presented demonstrate that overexpressing NF2 in a mouse melanoma cell line changes some of the major properties of these cells. In our study, we present the results of immunostaining the cells with an antibody specific for vinculin. Vinculin is a membrane-cytoskeletal protein found in focal adhesion plaques that is involved in the linkage of integrin adhesion molecules to actin cytoskeleton. The differences in the patterns observed in the B16F10-M cell line as compared to the control cells, would be predictive of changes in cell motility, focal adhesion, cell to cell contacts and regulation of signal transduction. Furthermore, the changes in the patterns of staining with an antibody specific for the heparan sulfate proteoglycan suggest that the presence of more Merlin molecules changes the cell surface organization of the cells. HSGAGs, along with the structural proteins, are key components of the cell surface-extracellular matrix interface, regulating the activity of numerous growth factors/morphogens, and their receptors, chemokines and extracellular proteins (13).

The localization of Merlin as well as its tumor suppressor properties suggests that this protein might interact with the transmembrane proteins - receptors and/or structural proteins, thus changing the spatial and structural organization of the extracellular matrix. The data obtained for the binding of Merlin to CD44 and its activation indicate that it depends on the ability of CD44 receptor to bind to its ligand, hyaluronan (HA), a mucopolysaccharide that is part of the extracellular matrix, and suggest that the cell surface organization is directly linked to the basic characteristics of the cells (15). Results have been presented indicating that inhibition of CD44-HA interaction contributes to the tumor suppressor function of Merlin, suggesting that, at least in part, Merlin acts by negatively regulating CD44 function (16).

Furthermore, our results indicate that Merlin-overexpressing cells, when tested for motility and growth in soft agar assays, display different properties when compared to the control cells. The data from the experiments in vivo demonstrated that increase NF2 expression resulted in a repression of the oncogenic properties of the B16F10-M cells; the tumor growth was delayed as compared to the control cells. The most striking difference was observed in the induced tumor metastasis model - the presence of additional Merlin molecules almost completely eliminated the ability of the cells to metastasize in the lungs of the tested animals.

The process of metastasis requires attachment of the cancer cells and the invasion/growth in a distant location. It is a very complex sequence of events that is directly linked to the cell surface organization. The glycosaminoglycan, heparan sulfate (HS), is composed of alternating units of glucosamine and uronic acid, that are variously sulfated at different positions. Proteoglycans carrying HS chains are expressed ubiquitously at cell surfaces and in the cellular matrix. The heparan sulfate chains interact with many different proteins, including growth factors, receptors, chemokines and extracellular matrix proteins. It was important to investigate whether the presence of an increased number of Merlin molecules would alter the distribution and/or 
organization of the cell surface HSGAGs. It was hypothesized that the glycan coat can protect the tumor cells from immune surveillance on the one hand, as well as play an important role in the promotion of tumor cell metastasis, by specific glycans mediating adhesion to distant sites. The experiments with isolated material from the cell surface of B16F10-M cells as compared to the material obtained and purified from the control cells strongly indicate that the cell surface was 'restructured' by the increased expression of the NF2 tumor suppressor gene. The composition of the accessible HS chains for the two enzymes tested (HepI and HepIII) was different, as the fractions isolated from the overexpressing cells inhibited the process of metastasis when injected with the control cells. It is important also to note that the amount of the added HSGAG fraction $(25 \mu \mathrm{g} / \mathrm{kg})$ was very small.

In the published literature, HSGAG organization, distribution and structure are considered to be characteristics that are very important for the metastatic properties of the cells, although the direct data are sparse. The results presented herein provide evidence of the causal link between the structure and function of HSGAGs. It would be worthwhile as a next step to analyze the differences in the composition and structure of the cell surface HSGAG preparation from the $\mathrm{B} 16 \mathrm{~F} 10-\mathrm{M}$ and the $\mathrm{B} 16 \mathrm{~F} 10$ control cells. Such analysis would have significant implications for our knowledge about the structure activity relations of the cell surface HSGAGs, and possibly provide additional targets for the treatment of cancer.

\section{References}

1. Tikoo A, Varga M, Ramesh V, Gusella J and Maruta H: An anti-RAS function of neurofibromatosis type 2 gene product (NF2/Merlin). J Biol Chem 269: 23387-23390, 1994.

2. Xiao GH, Beeser A, Chernoff J and Testa JR: p21 activated kinase links Rac/Ded42 signaling to merlin. J Biol Chem 277: 883-886, 2002.

3. Xiao GH, Gallagher R, Shetler J, Skele K, Altimare DA, Pestell RG, Jhanwar S and Testa JR: The NF2 tumor suppressor gene product, Merlin, inhibits cell proliferation and cell cycle progression by repressing cyclin D1 expression. Mol Cell Biol 25: 2384-2394, 2005.
4. McClatchey AI and Giovannini M: Membrane organization and tumorigenesis - the NF2 tumor suppressor, Merlin. Genes Dev 19: 2265-2277, 2005.

5. Okada T, You L and Giancotti FG: Shedding light on Merlin's wizardry. Trends Cell Biol 17: 222-229, 2007.

6. Blackhall FH, Merry CLR, Davies EJ and Jayson GC: Heparin sulfate proteoglycans and cancer. Br J Cancer 85: 1094-1098, 2001.

7. Fuster MM and Esko JD: The sweet and sour of cancer: glycans as novel therapeutic targets. Nat Rev Cancer 5: 526-542, 2005.

8. Galcheva-Gargova Z, Qi YW, Geisler S, Zhidkova N, Nugent MA and Venkataraman G: Genetically engineered mouse melanoma and mouse Lewis lung carcinoma models. Int J Oncol 26: 1233$1239,2005$.

9. Echtermeyer F, Streit M, Wilcox-Adelman S, Saoncella S, Denhez F, Detmar M and Goetinck PF: Delayed wound repair and impaired angiogenesis in mice lacking syndecan-4. J Clin Invest 107: R9-R14, 2001.

10. Gutmann DH, Sherman L, Setor L, Haipek C, Lu KH and Hendrix M: Increased expression of the NF2 tumor suppressor gene product, merlin, impairs cell motility, adhesion and spreading. Hum Mol Genet 8: 276-275, 1999.

11. Lallemand D, Curto M, Saotome I, Giovannini M and McClatchey A: NF2 deficiency promotes tumorigenesis and metastasis by destabilizing adherens junctions. Genes Dev 17: 1090-1100, 2003.

12. Curto M, Cole BK, Lallemand D, Liu C-H and McClatchey A: Contact-dependent inhibition of EGFR signaling by NF2/ Merlin. J Cell Biol 177: 893-903, 2007.

13. Lindahl U: Heparan sulfate-protein interactions - a concept for drug design? Throm Haemost 98: 109-115, 2007.

14. Liu D, Shriver Z, Venkataraman G, Shabrawi Y and Sasisekharan R: Tumor cell surface heparan sulfate as cryptic promoters or inhibitors of tumor growth and metastasis. Proc Natl Acad Sci USA 99: 568-573, 2002.

15. Morrison H, Sherman LS, Legg J, et al: the NF2 tumor suppressor gene product, merlin, mediates contact inhibition growth through interactions with CD44. Genes Dev 15: 968980,2001

16. Bai Y, Liu Y-J, Wang H, Xu Y, Stajmenkovic I and Yu Q: Inhibition of the hyaluronan-CD44 interaction by merlin contributes to the tumor-suppressor activity of merlin. Oncogene 26: 836-850, 2007. 